\section{Study protocol: Prosthesis versus Active (ProAct) exercise program in patients with glenohumeral osteoarthritis - a multicenter, randomized controlled trial}

\author{
Josefine Beck LARSEN 1,2, Theis Muncholm THILLEMANN 1,2, Antti P LAUNONEN ${ }^{3}$, \\ Helle Kvistgaard ØSTERGAARD ${ }^{2,4}$, Thomas FALSTIE-JENSEN ${ }^{1}$, Aleksi REITO ${ }^{3,5}$, \\ Steen Lund JENSEN ${ }^{6,7}$, and Inger MECHLENBURG ${ }^{1,2}$
}

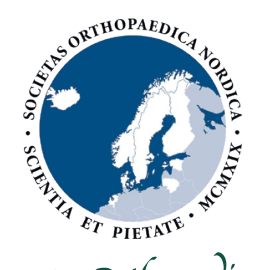

Acta Orthopaedica

\footnotetext{
${ }^{1}$ Department of Orthopaedic Surgery, Aarhus University Hospital, Aarhus N, Denmark; ${ }^{2}$ Department of Clinical Medicine, Aarhus University, Aarhus N, Denmark; ${ }^{3}$ Department of Orthopaedic Surgery, Tampere University Hospital, Tampere, Finland; ${ }^{4}$ Department of Orthopaedic Surgery, Viborg Regional Hospital, Viborg, Denmark; ${ }^{5}$ Faculty of Medicine and Health Technology, Tampere University, Tampere, Finland; ${ }^{6}$ Department of Orthopaedics, Aalborg University Hospital, Aalborg, Denmark; ${ }^{7}$ Department of Clinical Medicine, Aalborg University, Aalborg, Denmark

Correspondence: josefinebl@clin.au.dk

Submitted 2021-09-02. Accepted 2022-01-17-
}

Background and purpose - Total shoulder arthroplasty (TSA) and shoulder exercises are both effective treatments for reducing pain and improving function in glenohumeral osteoarthritis. However, the effectiveness of TSA has not been compared with non-surgical treatment in a randomized controlled trial. We will examine whether TSA followed by standard postsurgical rehabilitation is superior to a 12-week exercise program in patients with primary glenohumeral OA who are eligible for unilateral TSA.

Patients and methods - In this Nordic multicenter randomized controlled clinical trial, patients with glenohumeral osteoarthritis eligible for TSA will be allocated to either TSA followed by usual care or exercise only. The exercise intervention comprises 12 weeks of exercise with one weekly physiotherapist-supervised session. Based on the sample size calculation, the trial needs to include 102 patients.

Duration and outcome - Recruitment was initiated in April 2021 and is expected to be completed by the end of March 2024. Primary outcome is patient-reported quality of life, measured as total WOOS score 12 months after initiation of treatment. The key secondary outcomes include patient-reported pain intensity at rest and during activity; Disabilities of the Arm, Shoulder, and Hand score (DASH); the use of analgesics during the previous week; and adverse events.

Trial registration - The trial is approved by the Central Denmark Region Committee on Biomedical Research Ethics (Journal No 1-10-72-29-21) and by the Danish Data Protection Agency (Journal No 1-16-02-199-21). ClinicalTrials. gov Identifier: NCT04845074.
Anatomical total shoulder arthroplasty (TSA) is a wellestablished 3rd-line procedure for the treatment of glenohumeral osteoarthrosis $(\mathrm{OA})(\mathbf{1 , 2})$. In Denmark, the incidence of shoulder arthroplasty surgery nearly doubled between 2006 and $2015(3,4)$. Overall, the results after TSA have been good in terms of pain relief and functional outcome (3-6). However, complications after surgery and the subsequent risk for revision surgery are both associated with an inferior outcome (7-9).

It is generally accepted that the 1st- and 2nd-line treatment of glenohumeral OA is non-surgical, with the 1st-line treatment including activity modification and physiotherapy, and the 2nd-line treatment being pharmacological (1). To date, however, non-surgical treatment has never been compared with TSA surgery in patients who have moderate to severe primary glenohumeral OA. Several studies, including a Cochrane review (2), have suggested the need for trials that compare shoulder arthroplasty with non-surgical treatments, such as physiotherapist-supervised exercises (10-12). As selfdirected and physiotherapy-supervised exercises for patients with primary glenohumeral OA have been poorly described, we conducted a feasibility study in 2020 . The preliminary results from that study showed that a simple 12-week progressive exercise program is both feasible and safe for patients with glenohumeral OA or rotator cuff arthropathy who are eligible for shoulder arthroplasty surgery. Moreover, the shoulder function of patients improved, they experienced less pain, and 7 out of 20 patients postponed their shoulder surgery. It is therefore of value to conduct a well-powered RCT to compare the effects of TSA with a well-described exercise program in patients with moderate to severe glenohumeral OA. 
We will examine whether surgical treatment followed by standard postsurgical rehabilitation is superior to a 12-week exercise program in patients with moderate to severe primary glenohumeral OA who are eligible for unilateral shoulder arthroplasty. The primary outcome is the Western Ontario Osteoarthritis of the Shoulder (WOOS) index, measured 12 months after initiating the treatment. The primary hypothesis is that surgical intervention is superior to the exercise intervention at 12 months.

\section{Patients and methods}

\section{Study design}

The ProAct trial is a Nordic multicenter randomized controlled and investigator blinded trial. The study protocol is reported in accordance with the SPIRIT statement. The reporting will follow CONSORT guidelines.

\section{Setting and location}

Patients will be recruited from the Departments of Orthopaedic Surgery at Aarhus University Hospital, Aalborg University Hospital, Viborg Regional Hospital and Silkeborg Regional Hospital, Denmark, Oslo University Hospital, Norway, and Tampere University Hospital and Central Finland Central Hospital, Finland.

\section{Study participants and eligibility criteria}

Patients can be included if they are eligible for a standard anatomical total shoulder arthroplasty. In the term "standard" lies the understanding that the surgeons choose the standard prothesis with which they have experience. Stem length and fixation depends on the surgeon's choice. The use of new prothesis designs is not allowed, to reduce the risk of a learning curve bias.

\section{Inclusion criteria:}

1 . Patients $\geq 55$ years.

2. Moderate to severe primary glenohumeral OA (osteophyte larger than $3 \mathrm{~mm}$, according to Samilson and Prieto (13).

3. Eligible for surgery with standard TSA.

\section{Exclusion criteria:}

1. Need for bone graft or use of augmented glenoid component.

2. Previous shoulder fracture (fracture of the proximal humerus or glenoid fracture).

3. Planned other upper extremity surgery within 6 months.

4. Rheumatoid arthritis or other types of arthritis not diagnosed as primary glenohumeral OA.

5. Current cancer diagnosis receiving ongoing cancer treatment.

6. Neurological diseases affecting shoulder mobility (e.g., disability after previous stroke, multiple sclerosis, Parkinson's, Alzheimer's disease).
7. Other reasons for exclusion include mentally unable to participate or planned absence for more than 14 days in the first 3 months after baseline test.

8. Unable to communicate in the respective languages of the participating countries.

\section{Recruitment procedure}

The orthopedic surgeons of the attending hospitals will perform the inclusion, inform patients about the purpose of the study, and provide written information. Written informed consent will be obtained from all patients who are eligible and willing to participate. Patients can withdraw their consent without further reason at any time during the trial. Patients are not prohibited from seeking other treatment during the trial period.

\section{Randomization}

After baseline assessment, the patients will be randomized in a 1:1 ratio to either the TSA followed by usual care group (TSA group) or the physiotherapist-supervised exercise group (exercise group). A computer-generated list of random numbers will be set up in the Research Electronic Data Capture (REDCap; https://www.project-redcap.org/) randomize tool prior to the trial. The randomization will be stratified by site with randomly selected block sizes. Allocation concealment will be ensured, as the randomization will not be performed and revealed before the patient has been included in the trial. After randomization, a project coordinator will refer patients to surgery or to physiotherapistsupervised exercise.

\section{Data management}

Patient-reported outcomes will be entered directly into REDCap by the patients. If patients are not able to fill in the questionnaires on a computer, they will fill out paper versions instead and then a project coordinator will enter the data into REDCap. The raw dataset will be maintained in storage for 5 years after completion of the trial, with no access due to sensitive data. If required by the scientific journal in which the results are published, an anonymized patient-level dataset and corresponding statistical code will be made available. Otherwise, access to the completely anonymized patient-level dataset will be made available upon reasonable request.

\section{Observational cohort}

To evaluate the external validity, patients declining to participate in the trial will be invited to join a parallel prospective observational cohort using identical endpoints and identical primary and secondary patient-reported outcomes. Patients in the observational cohort will be offered standard surgical treatment with TSA. Written informed consent will be obtained from all patients who are willing to participate in the observational cohort. 


\section{Interventions}

TSA group

TSA will be performed through the deltopectoral approach, by experienced shoulder surgeons. Radiographs will be obtained postoperatively before discharge and at least at 3 and 12 months. Patients will commence postsurgical rehabilitation as usual. The patients will be instructed to use a sling for a minimum of 2 weeks, 24 hours a day. Patients are allowed to remove the sling when dressing, showering, and doing exercises. During the first 6 weeks after surgery, patients are allowed to move their shoulder passively within 0 degrees of external rotation and 90 degrees of flexion. From week 2, active flexion is allowed, and patients can use their arm for easy activities of daily living with their elbow fixed to their side. All patients will receive a written rehabilitation plan and will be instructed by a physiotherapist in postsurgical exercises. 6 weeks after surgery, patients are allowed to move their arm actively within their comfort zone and to slowly increase the exercise load. Those patients in the TSA group who decline surgery after randomization will be retained in the trial and asked to participate in the follow-up assessments.

\section{Exercise group}

The exercise group will attend a 12-week exercise program with 1 weekly physiotherapist-supervised session supplemented by 2 weekly sessions of home-based exercises. The utilization of a predefined training protocol describing the procedures and content of each session ensures the uniformity and standardization of the intervention. The exercise program consists of 2 warm-up exercises and 5 exercises that target shoulder range of motion, rotator cuff strength, and postural improvement (see Supplementary material). The exercise intervention is developed and described in accordance with the Consensus on Exercise Reporting Template (CERT) (14). The first supervised exercise session will be individual, and thereafter the exercises can be conducted in groups of 2-4 patients. To monitor adherence to the exercise intervention, patients will be asked to keep a training diary.

After completing the exercise program, the patients will have the possibility of attending an appointment with a shoulder surgeon at 3 months' follow-up. Should a patient experience any unsatisfactory effects, the patient may crossover to the TSA group, and the reasons for crossover will be registered. If patients choose to continue in the exercise group, they will receive 4 individual physiotherapist-supervised exercise booster sessions at $4,6,8$, and 10 months after initiating the exercise program as a means to maintain the effect (15).

\section{Outcomes}

Outcome assessments will be performed at baseline, 3 months, and 12 months' follow-up (from initial surgical/non-surgical treatment). An assessor, blinded to group allocation, will collect all the baseline measurements. At the subsequent out-

\section{Assessments and procedures}

\begin{tabular}{|c|c|c|c|c|c|c|}
\hline Factor & $\mathrm{BL}$ & $3 \mathrm{~m}$ & $1 \mathrm{y}$ & $2 y$ & $5 y$ & $10 y$ \\
\hline \multicolumn{7}{|l|}{ Baseline characteristics } \\
\hline Sex & $\mathrm{x}$ & & & & & \\
\hline Age & $\mathrm{x}$ & & & & & \\
\hline Height & $\mathrm{x}$ & & & & & \\
\hline Weight & $\mathrm{x}$ & & & & & \\
\hline Hand dominance & $x$ & & & & & \\
\hline Duration of shoulder symptoms & $x$ & & & & & \\
\hline Marital status & $x$ & & & & & \\
\hline Educational level & $\mathrm{x}$ & & & & & \\
\hline Employment status & $\mathrm{x}$ & & & & & \\
\hline Alcohol intake & $x$ & & & & & \\
\hline Smoking behaviors & $x$ & & & & & \\
\hline Comorbidities & $x$ & & & & & \\
\hline \multicolumn{7}{|l|}{ Surgery information } \\
\hline \multicolumn{7}{|l|}{$\begin{array}{l}\text { Radiography and } \\
\text { surgeon appoint }\end{array}$} \\
\hline Surgery report & \multirow{2}{*}{\multicolumn{6}{|c|}{$x^{x}$}} \\
\hline CT & & & & & & \\
\hline Radiography ${ }^{a}$ & & $x$ & $x$ & & & \\
\hline \multicolumn{7}{|l|}{ Patient-reported outcomes } \\
\hline WOOS b & $\mathrm{x}$ & $\mathrm{x}$ & $\mathrm{x}$ & $\mathrm{x}$ & $\mathrm{x}$ & $\mathrm{X}$ \\
\hline $\mathrm{DASH}^{\mathrm{c}}$ & $x$ & $x$ & $x$ & $x$ & $x$ & $x$ \\
\hline$E Q-5 D-5 L^{d}$ & $x$ & $x$ & $x$ & $\mathrm{X}$ & $x$ & $x$ \\
\hline $\mathrm{iPCQ}^{\mathrm{e}}$ & & $\mathrm{x}$ & $\mathrm{X}$ & & & \\
\hline VAS $^{f}$ & $x$ & $x$ & $x$ & $x$ & $\mathrm{x}$ & $\mathrm{X}$ \\
\hline \multicolumn{7}{|l|}{ Physical activity } \\
\hline Tri-axial accelerometry & $\mathrm{x}$ & & $x$ & & & \\
\hline \multicolumn{7}{|l|}{ Treatment-related variables } \\
\hline Adverse events $\mathbf{g}$ & & $\mathrm{x}$ & $\mathrm{x}$ & $\mathrm{X}$ & $\mathrm{x}$ & $\mathrm{x}$ \\
\hline Serious adverse events $g$ & & $x$ & $x$ & & & \\
\hline Training compliance & & $\mathrm{x}$ & & & & \\
\hline \multicolumn{7}{|l|}{$\begin{array}{l}\text { Pain before exercise and at the } \\
\text { end of exercise for the exercise } \\
\text { group using NRS }\end{array}$} \\
\hline Other shoulder-related treatments & $x$ & $x$ & $x$ & & & \\
\hline Analgesic consumption last week & $\mathrm{X}$ & $\mathrm{x}$ & $\mathrm{x}$ & $\mathrm{X}$ & $\mathrm{x}$ & $\mathrm{x}$ \\
\hline Crossover & & $x$ & $x$ & $\mathrm{X}$ & $x$ & $X$ \\
\hline
\end{tabular}

$\mathrm{BL}=$ baseline, $\mathrm{S}=$ surgery, $\mathrm{m}=$ months. $\mathrm{y}=$ years.

a Radiography at 3 months for the surgical group only to assess adverse events.

b Western Ontario Osteoarthritis of the Shoulder index (WOOS).

c Disabilities of the Arm, Shoulder and Hand (DASH).

d European Quality of life 5 Dimensions with 5 Levels (EC-5D-5L).

e Productivity Costs Questionnaire (iPQC), only in Denmark.

f Visual Analogue Scale will be at rest, during activity, and at night.

g Described in "Adverse events".

h Numeric Rating Scale will be used at the supervised exercise sessions to determine pain at rest before and after the session.

come assessments, patients will complete the questionnaires from home (online or via letter). At 12 months' follow-up, the patients will complete all questionnaires from home and attend a research visit at the hospital, where the site's research personnel will mount accelerometers on the upper arms to enable the measurement of physical activity. The patients will be instructed to wear a T-shirt to cover any surgical scar. All patients will be sent shoulder-related questionnaires at 2, 5 , and 10 years after inclusion in the trial. An overview of assessed baseline characteristics and outcomes is presented in the Table. 


\section{Primary outcome}

The primary outcome is the total WOOS score at 12 months' follow-up. It is a valid, reliable, and responsive outcome measurement of the shoulder-related quality of life of patients with OA described by 4 domains: (1) physical symptoms; (2) sport, recreation, and work; (3) lifestyle; and (4) emotions (16). The questionnaire consists of 19 items using a visual analog scale (VAS). Each item has a possible score ranging from 0 to 100 , leading to a total WOOS score ranging from 0 to 1900 , with 0 being the best. Raw scores can be converted to a percentage of the maximum score $(0-100,100=$ best $)$. The WOOS score has been validated in the Danish population on patients with glenohumeral OA treated with shoulder arthroplasty (16).

\section{Secondary outcomes}

The secondary outcomes include WOOS at the other time points; Disabilities of the Arm, Shoulder and Hand (DASH) (17); patient-reported pain intensity at rest, during activity, and nightly pain using the $100 \mathrm{~mm}$ VAS; the use of analgesics during the last week (paracetamol, NSAID, opioids); adverse events.

\section{Serious adverse events}

Serious adverse events (SAE) are defined as embolism (cardiac or brain), death, liver, and renal failure resulting in the need for hospitalization or death. Patients in the TSA group will be monitored for serious adverse events during the first 4 weeks after discharge.

\section{Adverse events}

Adverse events or harms will be defined as any unintended and unfavorable sign, symptom, or disease, resulting in contact with the healthcare system irrespective of a causal relationship with the intervention and outcome assessments. Serious and unexpected adverse events will be monitored during the period from initial inclusion until 12 months' follow-up and will be reported according to the recommendations given by the Consort Group:

1. For the surgical intervention group, the primary focus will be on postoperative infections, instability, periprosthetic fracture, or loosening of 1 or more of the arthroplasty components.

2. For the non-surgical group, the primary focus will be on exercise-related injuries. For example, persistent pain, fatigue, bursitis, low back pain, and edema (18).

At the halfway stage of the trial $(50 \%$ of patients recruited in the trial), an independent auditing committee will evaluate the complication rates and correlate them to the expected rates published in the available literature. An unexpected high rate of complications in either group will be reported to the project group, who will then decide whether the randomization needs to be halted or whether the study can continue. No formal data monitoring committee will be composed as SAEs of both interventions are well known $(\mathbf{5 , 7 , 1 8 - 2 1 )}$. Any SAEs occurring from baseline to 12 months' follow-up will be discussed by the author group. No interim analysis will be performed.

\section{Exploratory outcomes}

Exploratory outcomes include evaluation of shoulder activity using tri-axial accelerometers (Axivity, Newcastle upon Tyne, UK) at baseline and at 12 months' follow-up (22); patientreported pain intensity at rest (exercise group) before and after the exercise intervention using the Numeric Rating Scale (NRS); health-related quality-of-life measured with EuroQol 5-dimension (EQ-5D-5L); the Productivity Costs Questionnaire (iPCQ) but only in Denmark (23); Patient acceptable symptom state; number of TSAs in the exercise group at longterm follow-up (2, 5, and 10 years); adherence to supervised exercise sessions from baseline to 3 months (exercise group); adherence to unsupervised exercise (exercise group).

\section{Sample size}

The sample size calculation is based on WOOS data from the Danish Shoulder Arthroplasty Register and on end scores obtained from the feasibility study. The median WOOS score 1 year after surgery for patients with glenohumeral OA treated with TSA is 85 points (Danish Shoulder Arthroplasty Registry [DSR] 2020). The assumed SD was 27, calculated from the interquartile range (IQR). After completing the exercise intervention in the feasibility study, the WOOS score was a mean 67 (SD 22). With a 5\% level of significance and a sample size of 78 patients, the study will have $80 \%$ power to detect an 18-point difference in the end scores between the surgical and exercise group. Assuming a 30\% dropout rate, the total number of patients needed is 102 .

\section{Statistics}

Continuous variables will be reported using means and standard deviation (SD). 95\% confidence intervals (CI) for proportions will be calculated using the Wilson method. Binary and categorical variables will be reported using counts (\%). A 2 -sided alpha level of 0.05 will be considered as statistically significant.

\section{Primary analysis}

Primary outcome is the total WOOS at 12 months. The primary analysis will follow the intention-to-treat principle and includes all randomized patients regardless of actual treatment.

The primary comparison in WOOS between groups will be done using a linear mixed model. Intervention group and time (3 months, 12 months) will be included as fixed effect and patient as random. Baseline score, age, sex, and study site will also be included as fixed covariates. Interaction between study group and time will be included in the model to estimate treatment effect at each time point. CIs are estimated for each time point. Due to the repeated mixed model analysis no missing data imputation will be done. 
Secondary analysis

Analysis for patient-reported DASH, with pain intensity at 3 and 12 months, will be analyzed similar to WOOS. For binary variables (i.e., the use of analgesics and adverse events) we use logistic regression to estimate treatment effects. For each binary outcome an average marginal difference in proportions of outcomes between study groups is estimated from a logistic model. Baseline score, age, sex, and study site will be used as covariates.

Sensitivity and exploratory analysis will be performed with the purpose to test the robustness of the intention-to-treat analysis including a per-protocol analysis for the primary outcome, excluding patients who had poor adherence to the exercise intervention and crossover patients. Poor adherence is defined as participating in less than $70 \%$ of the supervised exercise sessions. Crossover patients are defined as patients allocated to the exercise group who undergo TSA during follow-up. Lastly an as-treated analysis will be performed in which patients will be analyzed based on their adherence to the randomized treatment expecting 4 groups: (1) patients randomized to TSA and receiving TSA, (2) patients randomized to exercise without undergoing TSA in the follow-up period, (3) patients randomized to exercise undergoing TSA in the follow-up period, (4) patients randomized to TSA not undergoing TSA in the follow-up period.

Data analyses will be conducted according to a pre-developed statistical analysis plan made publicly available prior to inclusion of the final patient. All statistical analysis will be undertaken using the latest STATA software (StataCorp LP, College Station, TX, USA).

\section{Ethics, registration, dissemination, funding, and con- flicts of interest}

Before inclusion, written informed consent in accordance with the Declaration of Helsinki II will be obtained from all patients. All data and information collected regarding this trial will be treated confidentially by researchers and staff connected to the trial.

The trial is approved by the Central Denmark Region Committee on Biomedical Research Ethics (Journal No 1-10-7229-21) and by the Danish Data Protection Agency (Journal No 1-16-02-199-21). The trial will follow Good Clinical Practice (GCP) guidelines. Monitoring of the trial by the GCP unit is not required. Results from this trial will be published in international peer-reviewed scientific journals regardless of whether the results are positive, negative, or inconclusive. Authorship eligibility will be based on the recommendations from the International Committee of Medical Journal Editors. All authors declare no conflicts of interest.

Any important protocol amendments will be addressed in the primary trial manuscript and registered at ClinicalTrials. gov and reported to the Central Denmark Region Committee on Biomedical Research Ethics.

The trial is supported by the Health Research Foundation of Central Denmark Region, Aarhus University, the Danish
Rheumatism Association, the Association of Danish Physiotherapists, the Health Foundation (Helsefonden), the Hartmann's Foundation, and the Emil Hertz Foundation in Denmark. The foundations had no role in the planning of the trial and provided only financial support.

\section{Study start and duration}

Recruitment commenced in April 2021 and final patient recruitment is expected to be completed by the end of March 2023.

\section{Discussion}

Considering the increase in the number of shoulder arthroplasties performed due to glenohumeral OA, it is striking that the effectiveness of TSA has not previously been compared with exercise in an RCT. This is the 1st study to evaluate the effectiveness of surgical treatment compared with non-surgical treatment in patients with glenohumeral OA who are eligible for TSA. By highlighting the benefits and harms of surgical and non-surgical treatment approaches, the PROACT trial will provide valuable evidence to patients and their relatives, surgeons, physiotherapists, and decision-makers.

There are some potential limitations in the trial. 1st, it is not possible to blind patients, participating surgeons, or physiotherapists supervising the exercise intervention, which may affect how patients report the outcomes. 2nd, low recruitment is a potential major limitation when conducting a trial that involves randomizing surgical or non-surgical interventions. However, the possibility of low recruitment rates is reduced by the multicenter setup. Furthermore, eligible patients who decline participation in the trial will be invited to participate in a prospective cohort study to evaluate the external validity of the results. There is a risk of crossover if the non-surgical treatment results in insufficient pain relief. However, in an earlier feasibility study, 7 out of 20 included patients postponed their scheduled surgery, and therefore it is likely that a substantial number of patients will remain in the non-surgical group during the follow-up period.

The strengths of the trial are the use of methods that include a multicenter, assessor-blinded, randomized controlled design, protocol publication, and blinded interpretation. The interventions are conducted in a clinical setting, and thus enhance the potential future implementation of the treatments in the healthcare system. The exercise protocol has been developed based on the available evidence on patients with glenohumeral osteoarthritis, rotator cuff arthropathy, and massive rotator cuff tears. It is a well-defined exercise protocol that is simple to implement for therapists and simple for patients to conduct at home. Finally, in the feasibility study conducted prior to this trial, patients were involved in the setting of priorities for primary and secondary outcomes and gave important feedback on how to organize the exercise intervention. 
All authors are members of the PROACT study group. JBL, TMT, AL, and IM designed the trial. JBL is the primary investigator and main author. JBL, TMT, AL, and IM wrote the protocol, and HKØ, TFJ, AR, and SLJ revised the protocol. All authors approved the final version.

Acta thanks Jos JAM van Raaij for help with peer review of this protcol.

1. Roos E M, Juhl C B. Osteoarthritis 2012 year in review: rehabilitation and outcomes. Osteoarthritis Cartilage 2012; 20(12): 1477-83. doi: https://doi.org/10.1016/j.joca.2012.08.028.

2. Craig R S, Goodier H, Singh J A, Hopewell S, Rees J L. Shoulder replacement surgery for osteoarthritis and rotator cuff tear arthropathy. Cochrane Database Syst Rev 2020; 4: CD012879. doi: 10.1002/14651858. CD012879.pub2.

3. Rasmussen J V, Amundsen A, Sorensen A K B, Klausen T W, Jakobsen J, Jensen S L, et al. Increased use of total shoulder arthroplasty for osteoarthritis and improved patient-reported outcome in Denmark, 2006-2015: a nationwide cohort study from the Danish Shoulder Arthroplasty Registry. Acta Orthop 2019; 90(5): 489-94. doi: 10.1080/17453674.2019.1633759.

4. Danish Shoulder Arthroplasty Registry (DSR). Annual Report 2020 [cited 2021, February 24]. Available from: https://www.sundhed.dk/content/cms/3/4703_dsr_aarsrapport2020_offentliggoerelse.pdf.

5. Rasmussen J V, Polk A, Brorson S, Sørensen A K, Olsen B S. Patient-reported outcome and risk of revision after shoulder replacement for osteoarthritis: 1,209 cases from the Danish Shoulder Arthroplasty Registry, 2006-2010. Acta Orthop 2014; 85(2): 117-22. doi: 10.3109/17453674.2014.893497.

6. Rasmussen J V, Olsen B S. The Danish Shoulder Arthroplasty Registry. Obere Extrem 2019; 14(3): 173-8. doi: 10.1007/s11678-019-0524-2.

7. Craig R S, Lane J C E, Carr A J, Furniss D, Collins G S, Rees J L. Serious adverse events and lifetime risk of reoperation after elective shoulder replacement: population based cohort study using hospital episode statistics for England. BMJ 2019; 364: 1298. doi: 10.1136/bmj. 1298.

8. Shields E, Wiater J M. Patient outcomes after revision of anatomic total shoulder arthroplasty to reverse shoulder arthroplasty for rotator cuff failure or component loosening: a matched cohort study. J Am Acad Orthop Surg 2019; 27(4): e193-e8. doi: 10.5435/jaaos-d-17-00350.

9. Ravi V, Murphy R J, Moverley R, Derias M, Phadnis J. Outcome and complications following revision shoulder arthroplasty. Bone Jt Open 2021; 2(8): 618-30. doi: 10.1302/2633-1462.28.Bjo-2021-0092.R1.

10. Izquierdo R, Voloshin I, Edwards S, Freehill M Q, Stanwood W, Wiater J M, et al. Treatment of glenohumeral osteoarthritis. J Am Acad Orthop Surg 2010; 18(6): 375-82. doi: 10.5435/00124635-20100600000010 .

11. Singh J A, Sperling J, Buchbinder R, McMaken K. Surgery for shoulder osteoarthritis. Cochrane Database Syst Rev 2010(10). doi: 10.1002/14651858.CD008089.pub2.

12. Floyd S B, Chapman C G, Shanley E, Ruffrage L, Matthia E, Cooper $\mathbf{P}$, et al. A comparison of one-year treatment utilization for shoulder osteoarthritis patients initiating care with non-orthopaedic physicians and orthopaedic specialists. BMC Musculoskelet Disord 2018; 19(1): 349. doi: 10.1186/s12891-018-2268-3.

13. Samilson R L, Prieto V. Dislocation arthropathy of the shoulder. J Bone Joint Surg Am 1983; 65(4): 456-60. PubMed PMID: 6833319.

14. Slade S C, Dionne C E, Underwood M, Buchbinder R, Beck B, Bennell K, et al. Consensus on Exercise Reporting Template (CERT): Modified Delphi Study. Phys Ther 2016; 96(10): 1514-24. doi: 10.2522/ ptj.20150668.

15. Taul-Madsen L, Kjeldsen T, Skou S T, Mechlenburg I, Dalgas U. Exercise booster sessions as a means to maintain the effect of an exercise-intervention: a systematic review. Phys Ther Rev 2021:1-11. doi: 10.1080/10833196.2021.1988816

16. Rasmussen J V, Jakobsen J, Olsen B S, Brorson S. Translation and validation of the Western Ontario Osteoarthritis of the Shoulder (WOOS) index: the Danish version. Patient Relat Outcome Meas 2013; 4:49-54. doi: 10.2147/PROM.S50976.

17. Lindenhovius A, Buijze G, Kloen P, Ring D. Correspondence between perceived disability and objective physical impairment after elbow trauma. J Bone Joint Surg Am 2008; 90: 2090-7. doi: 10.2106/JBJS.G.00793.

18. Niemeijer A, Lund H, Stafne S N, Ipsen T, Goldschmidt C L, Jørgensen $\mathbf{C} \mathbf{T}$, et al. Adverse events of exercise therapy in randomised controlled trials: a systematic review and meta-analysis. Br J Sports Med 2020; 54(18): 1073. doi: 10.1136/bjsports-2018-100461.

19. Boileau P. Complications and revision of reverse total shoulder arthroplasty. Orthop Traumatol Surg Res 2016; 102(1 Suppl.): S33-43. doi: 10.1016/j.otsr.2015.06.031.

20. Rasmussen J V, Hole R, Metlie T, Brorson S, Aarimaa V, Demir Y, et al. Anatomical total shoulder arthroplasty used for glenohumeral osteoarthritis has higher survival rates than hemiarthroplasty: a Nordic registrybased study. Osteoarthritis Cartilage 2018; 26(5): 659-65. doi: 10.1016/j. joca.2018.02.896.

21. Rasmussen J V, Jakobsen J, Brorson S, Olsen B S. The Danish Shoulder Arthroplasty Registry: clinical outcome and short-term survival of 2,137 primary shoulder replacements. Acta Orthop 2012; 83(2): 171-3. doi: 10.3109/17453674.2012.665327.

22. Sliepen M, Lipperts M, Tjur M, Mechlenburg I. Use of accelerometer-based activity monitoring in orthopaedics: benefits, impact and practical considerations. EFORT Open Rev 2020; 4(12): 678-85. doi: 10.1302/2058-5241.4.180041.

23. Bouwmans C, Krol M, Severens H, Koopmanschap M, Brouwer W, Hakkaart-van Roijen L. The iMTA Productivity Cost Questionnaire: a standardized instrument for measuring and valuing health-related productivity losses. Value Health 2015; 18(6): 753-8. doi: 10.1016/j. jval.2015.05.009. 


\section{(O PROACT O) REACT}

\section{Exercise program to increase mobility and strength in the shoulder joint}

You should perform the exercise program 3 times a week. You are welcome to do the warm-up exercises every day, and you are recommended to be generally physically active in leisure activities that do not provoke known shoulder pain. For each exercise, there are two levels of difficulty, and you only need to perform the level that you have ticked-off in collaboration with the physiotherapist. It takes about 20 minutes to complete the shoulder exercises.

\section{You will be given 3 things:}

1. Home-training program, undertaken with advice from the physiotherapist

2. Elastic bands for the exercises ( +3 tips on how to give your elastic band good durability)

3. Exercise diary, which must be completed after each exercise session

At the last training session, you will be advised by the physiotherapist how to continue the training at home.

While performing the exercises, you should not have a pain score above 5 measured on a scale of $0-10$, where 10 is the worst imaginable pain. The pain should subside quickly (and should last no longer than 2 hours) after completing the exercises.

\begin{tabular}{|l|l|}
\hline Pain $\leq 2$ & Safe \\
\hline Pain $\leq 5$ & Acceptable \\
\hline Pain $>5$ & Risk \\
\hline
\end{tabular}


1. Pendulum: Let your arm hang down towards the floor and make 20 small circles, first 10 counterclockwise then 10 clockwise.
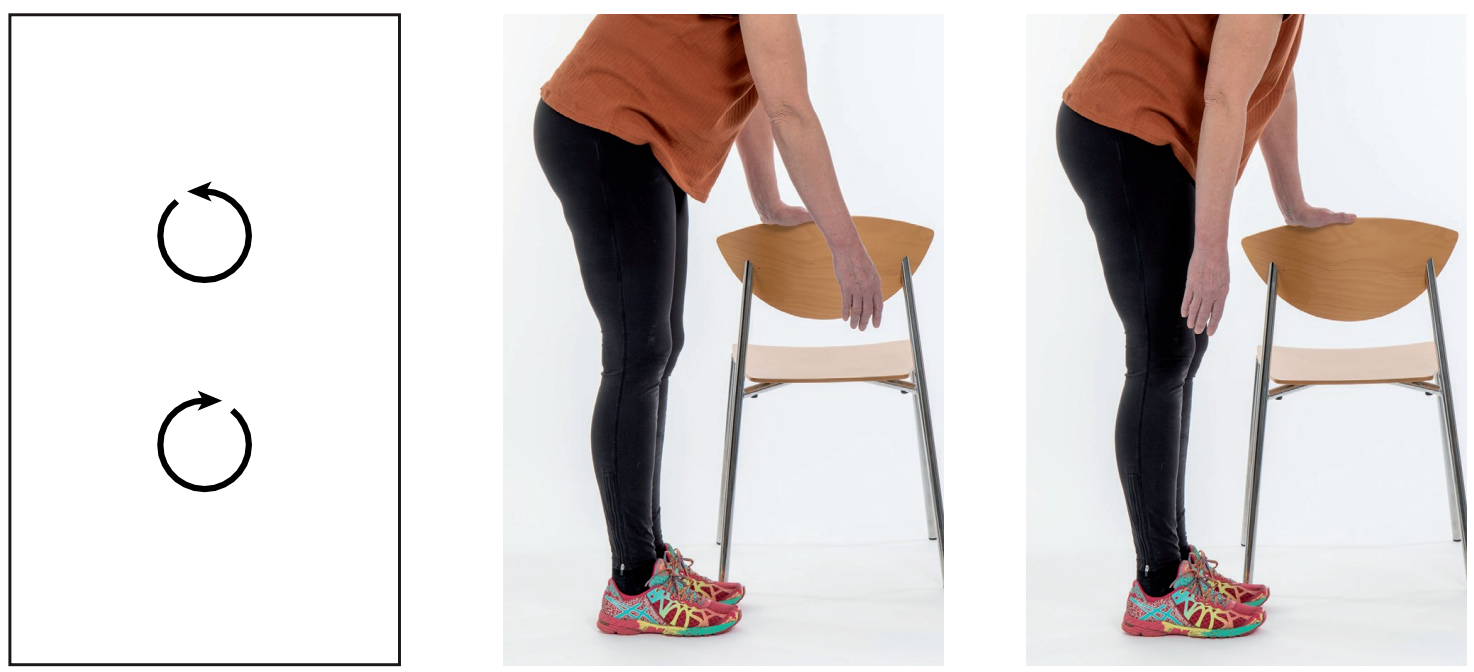

2. Table slides: Place a dry cloth on the table and move it back and forth 10 times, then in a large circle 10 times, and finally in figure of eight 10 times.
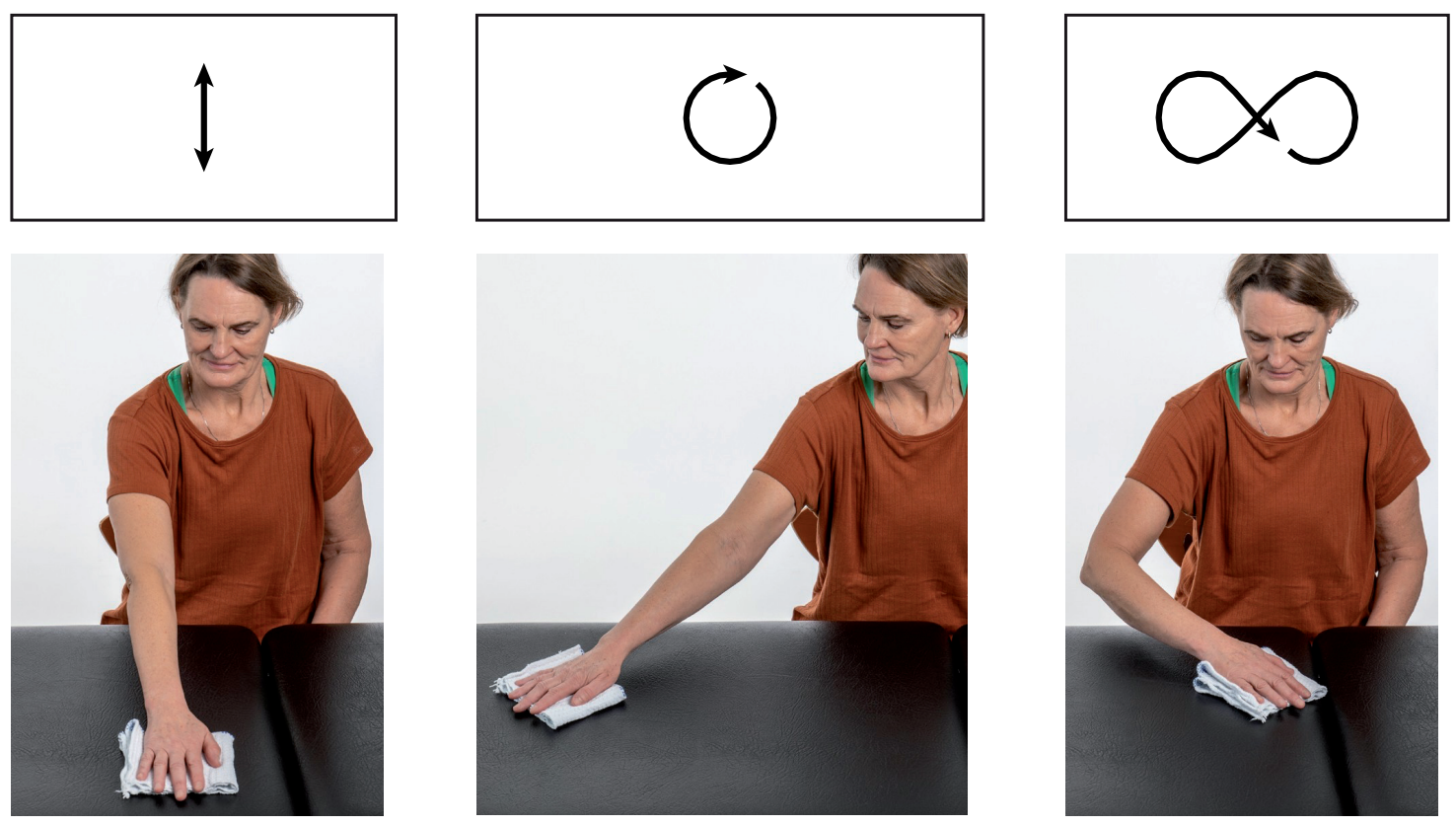
Elastic band color:

Set:

Repetitions:

Level B: Sit at a table with a cloth under your elbow. Keep the elbow bent at 90 degrees. Move the arm out to the side, without moving the position of the elbow. Gently return to starting position and repeat.
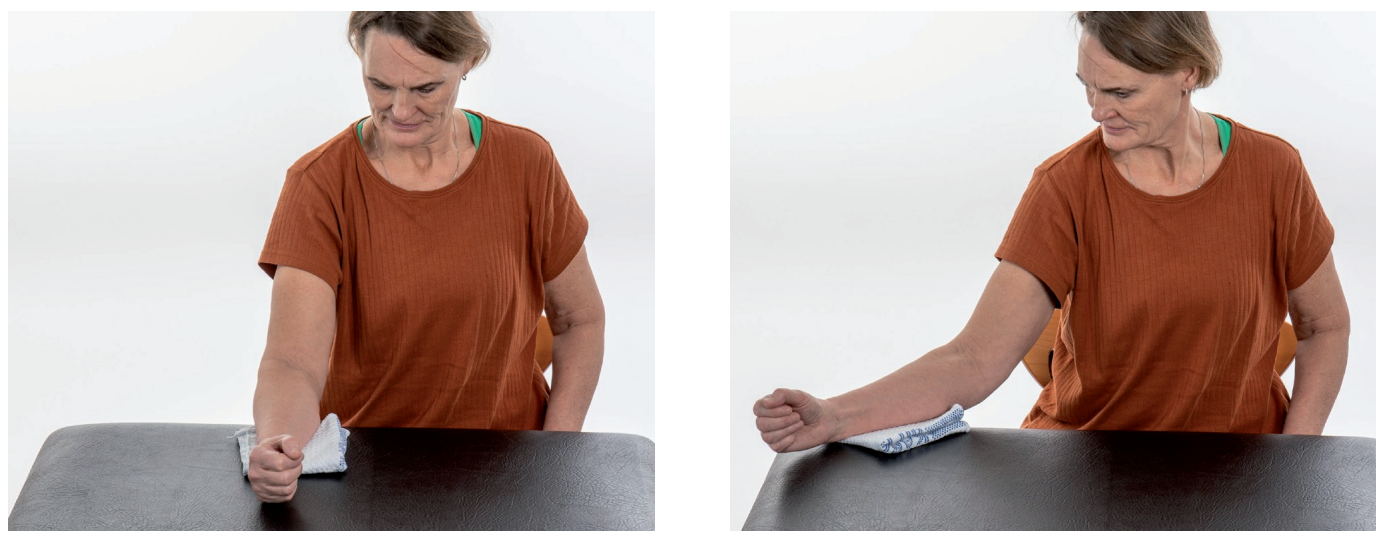

Level A: Secure the elastic band at waist level. Keep your elbow at your side, with a 90-degree bend in your elbow. Rotate your hand away from your body as far as you can without moving your elbow. Return to the starting position. Place a small towel between your elbow and body.
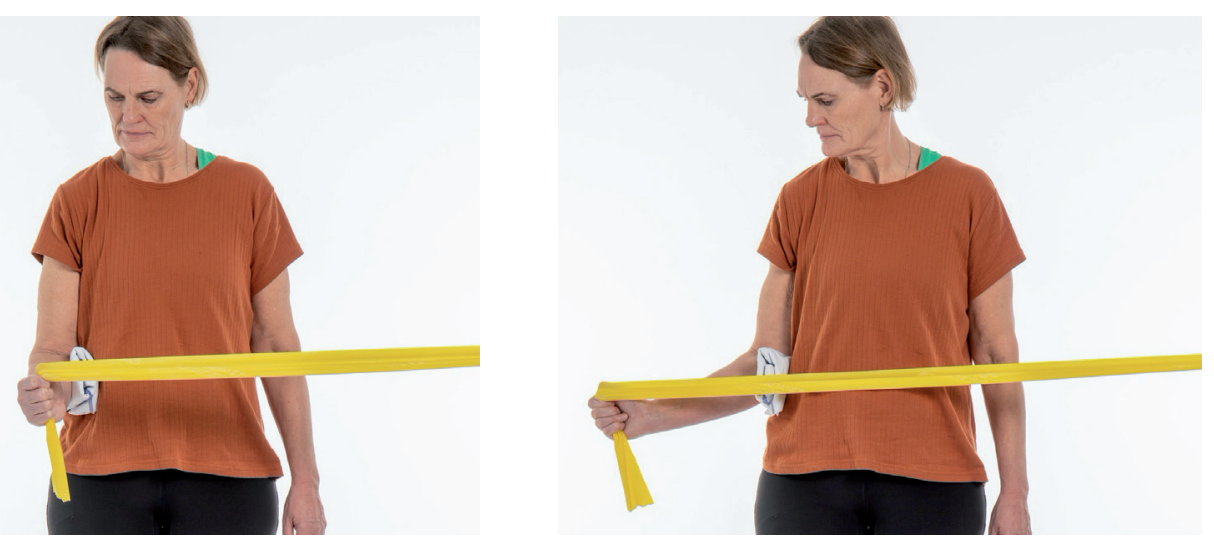
Elastic band color:

Set:

Repetitions:

Level B: Sit at a table with a cloth under your elbow. Keep the elbow bent at 90 degrees. Rotate your arm in towards your stomach, without moving your elbow. Gently return to starting position and repeat.
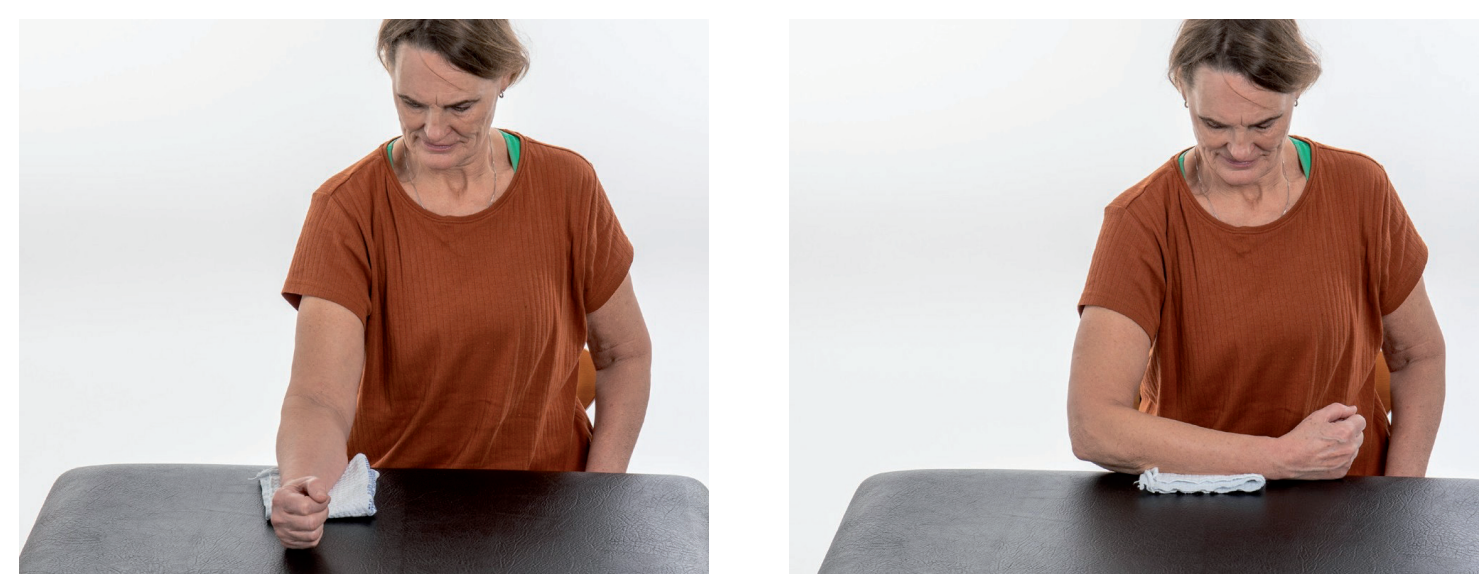

Level A: Secure the elastic band at waist level. Keep your elbow at your side, with a 90-degree bend in your elbow. Rotate your hand towards your stomach as far as you can without moving your elbow. Return to the starting position. Place a small towel between your elbow and body.
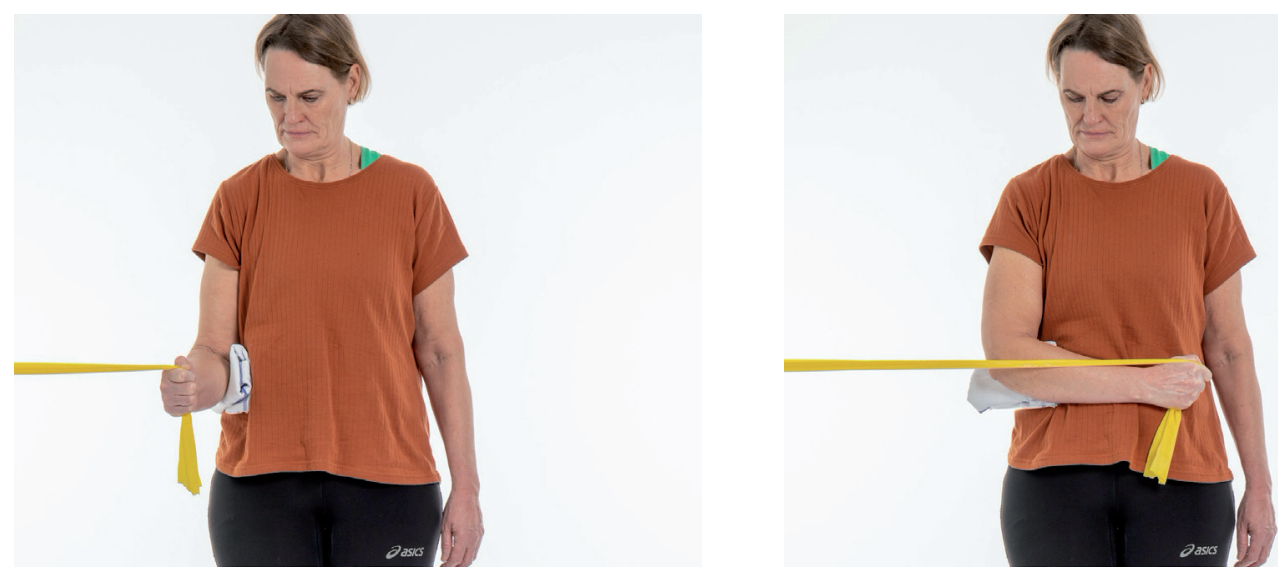
Elastic band color:

Set:

Repetitions:

Level B: Stand upright with your arms hanging along your body. Inhale while pulling the shoulder blades down towards the "back pocket", hold for 3 seconds. Gently return to starting position and repeat.
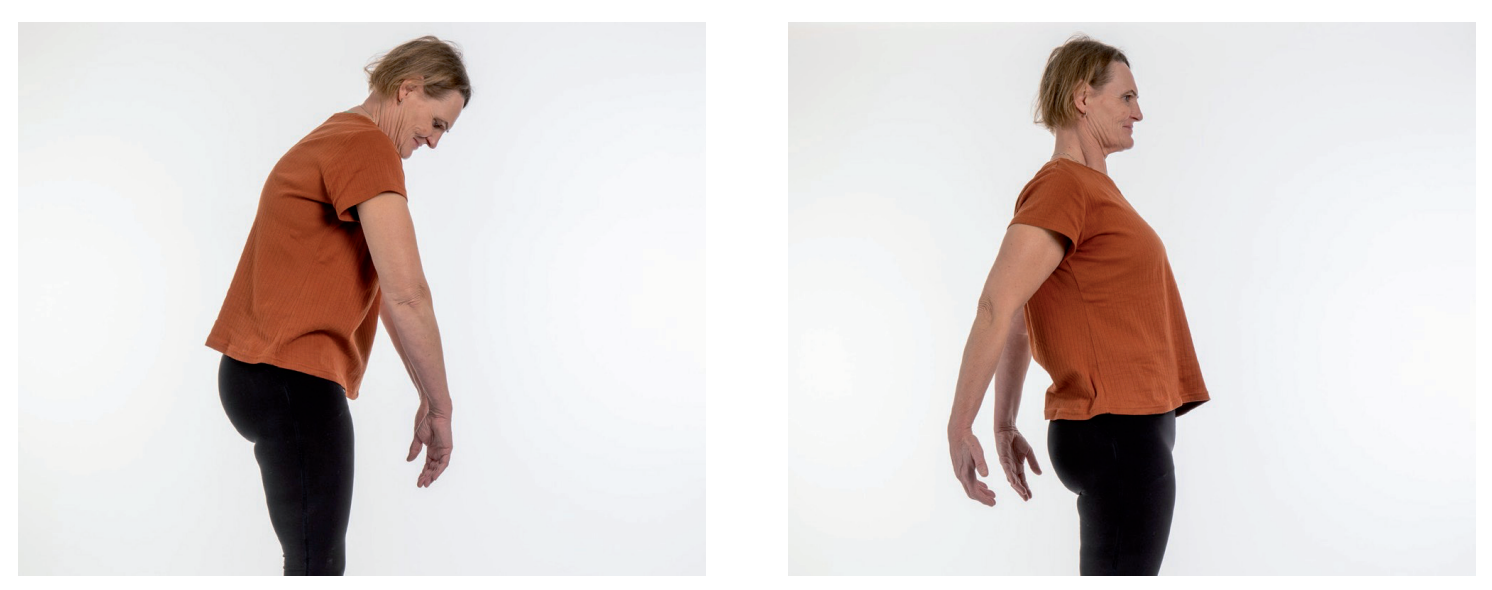

Level A: Secure the elastic band at waist level. Stand facing the elastic band attachment and one end of the band in each hand. Keep elbows straight and pull. Try to pull past your hips. Gently return to starting position and repeat. Be aware that your elbows are stretched throughout the movement.
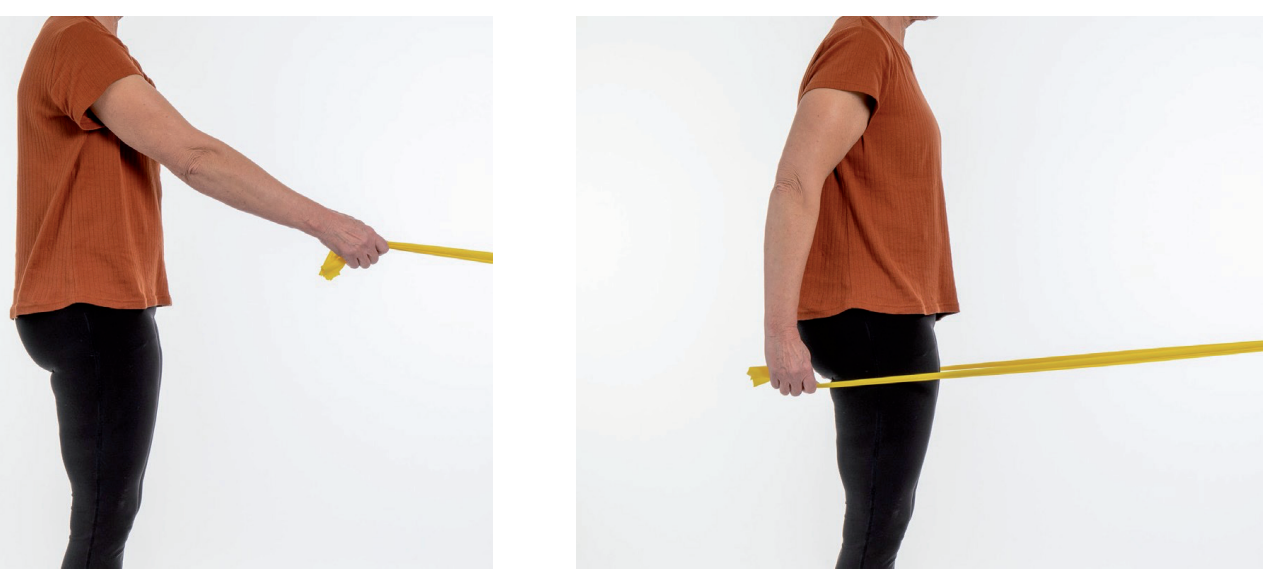
Elastic band color:

Set:

Repetitions:

Level B: Stand facing the wall with a cloth under your hand. The arm moves from chest height and upwards, then downwards in a big circle away from your body. Gently return to starting position and repeat.
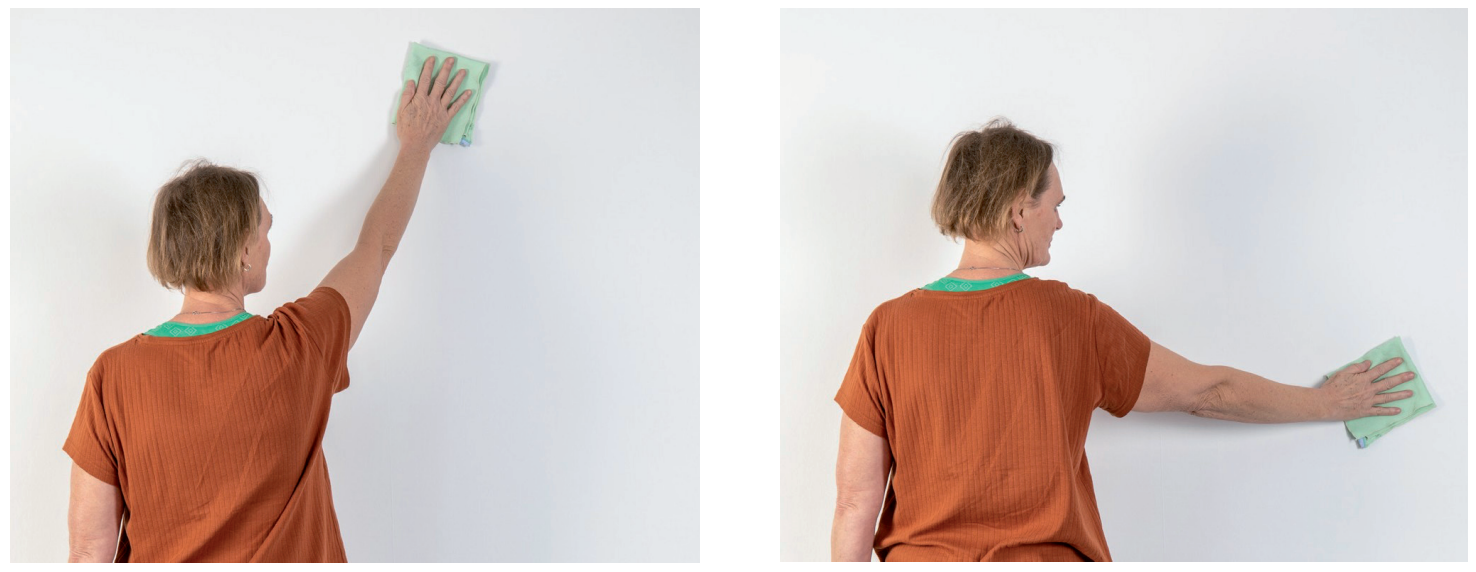

Level A: Secure the elastic band by standing with one foot on one end of the band. Hold the other end of the band in the opposite hand. Elevate your arm out to the side as high as you can. The elbow is slightly bent during the entire movement. Gently return to starting position and repeat.
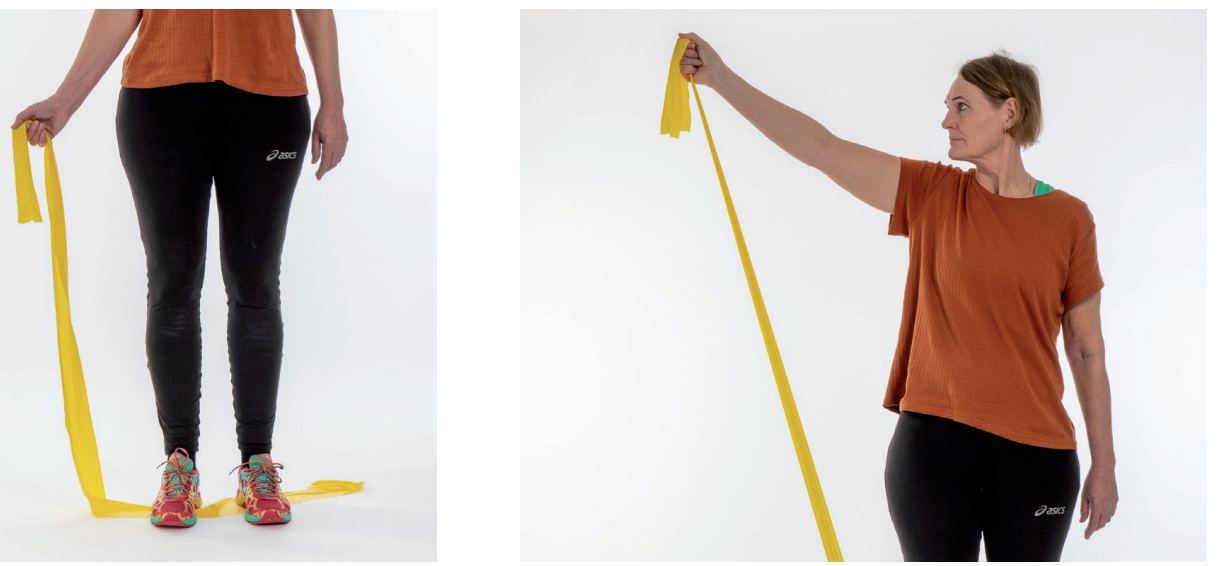
Elastic band color:

Set:

Repetitions:

Level B: Lie on your back with your arms down alongside your body. Lift your arms above your head. Gently return to starting position and repeat.
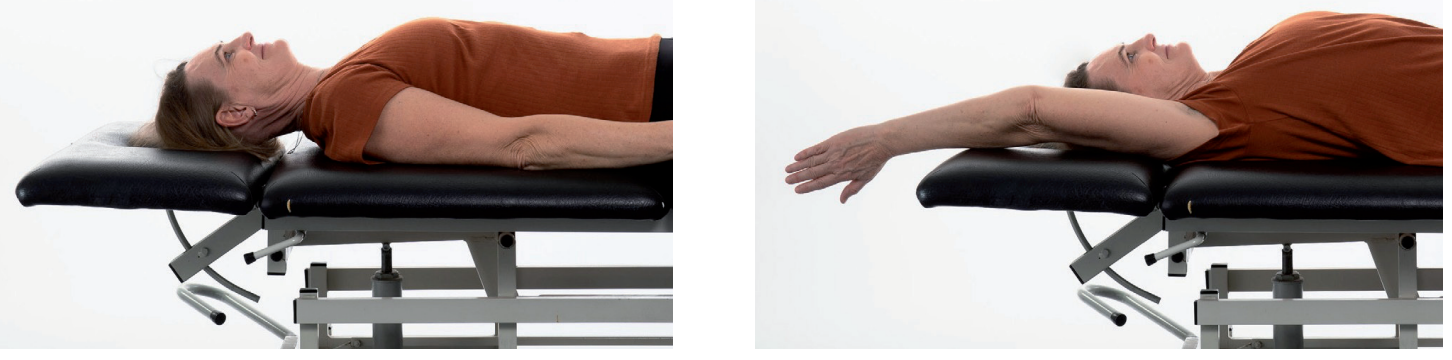

Level A: Secure the elastic band by standing with one foot on the middle of the band. Hold the elastic in front of the body with the thumbs pointing upwards. Pull the band up and forward in front of the body with outstretched arms as high as you can. Gently return to starting position and repeat.
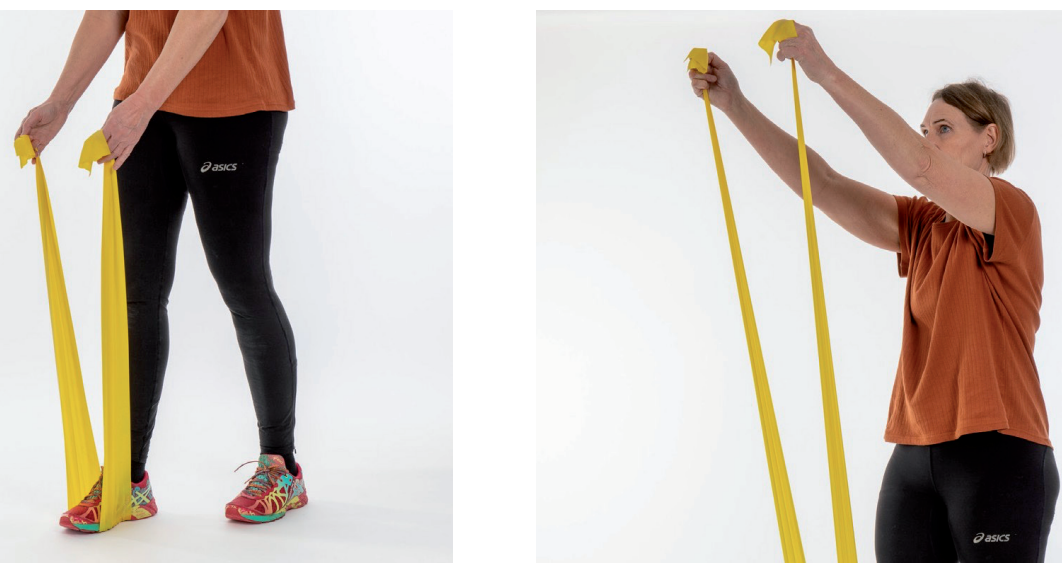
3 TIPS TO GIVE YOUR ELASTIC BAND GOOD DURABILITY

1. If the band is damp or wet, hang it to dry over a chair

2. Store the band dry and not in direct sunlight or at high heat

3. The band should be powdered occasionally with a little baby powder or ordinary talcum powder

Later

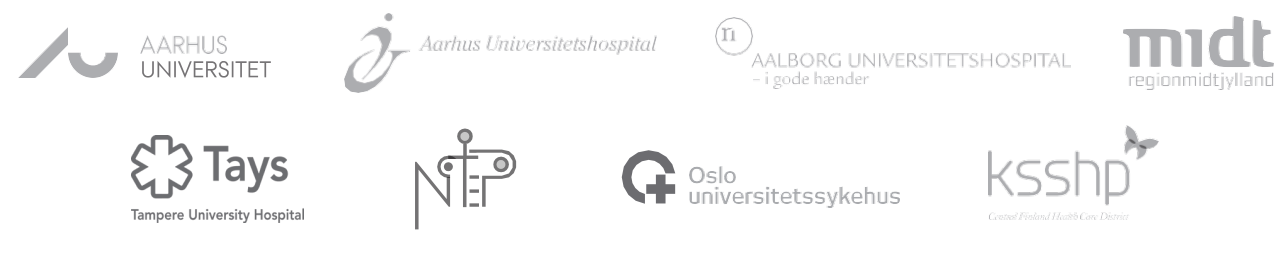

